\title{
Efeito de substratos agrícolas na produção de mudas de hortelã propa- gadas por estaquia
}

\author{
Dalva Paulus ${ }^{1,3}$; Eloi Paulus ${ }^{2}$ \\ ${ }^{1}$ USP/ESALQ, Depto. Eng. Rural, 13418-900 Piracicaba-SP;. ${ }^{2}$ C. postal 5066, 97105-120 Santa Maria-RS; ${ }^{3}$ End. para correspondência: \\ R. Dona Eugênia, 1430 apto. 08, 13416-101 Piracicaba-SP; dalvaufsmdeutch@yahoo.com.br
}

\section{RESUMO}

A utilização de substratos agrícolas, adequados a cada espécie vegetal, auxilia na obtenção de mudas e plantas com qualidade comercial. $\mathrm{O}$ experimento foi realizado na Universidade Federal de Santa Maria (RS) com objetivo de avaliar o efeito de substratos (organo-mineral, casca de arroz carbonizada + areia (1:1) e casca de arroz + solo (1:1)) para produção de mudas de hortelã (Mentha arvensis L.) adequadas ao cultivo no campo. O experimento teve duas fases: produção das mudas em ambiente protegido e desenvolvimento das plantas no campo. O delineamento experimental foi inteiramente casualizado, com três tratamentos e 15 repetições. Foram avaliados a altura de planta e número de folhas aos 7, 14, $21 \mathrm{e}$ 28 dias após transplante, índice de mortalidade, matéria fresca e seca 35 dias após o transplante. As mudas produzidas no substrato organomineral apresentaram os melhores resultados durante a fase de desenvolvimento a campo com relação à produção de matéria fresca $\left(7,07 \mathrm{~g}\right.$ planta $\left.^{-1}\right)$ e matéria seca $\left(2,40 \mathrm{~g}_{\text {planta }}{ }^{-1}\right)$. Tanto a mistura de casca de arroz carbonizada + solo ou areia também se mostraram apropriados para a produção de mudas de hortelã para o cultivo a campo.

Palavras-chave: Mentha arvensis, plantas medicinais, estaquia.

\begin{abstract}
Effect of agricultural substrates in the production of mint seedlings propagated through cuttings

The use of appropriated substrates for each vegetative species is important to obtain seedlings and plants with commercial quality. The experiment was carried out in Rio Grande do Sul State, Brazil. The effect of substrates (organic-mineral, carbonized rice husk + sand $(1: 1)$ and carbonized rice husk + soil (1:1)) was determined for the production of appropriated mint seedlings (Mentha arvensis L.) for field cultivation. The experiment was performed in two phases: production of the seedlings under protected environment and growth of the plants in field. The experimental design was a completely randomized design with three treatments and 15 replications. We evaluated the height and number of leaves at 7; 14;21 and 28 days after the transplant; index of mortality; fresh and dry matter, 35 days after transplanting date. The organic-mineral substrates presented the best results in relation to the production of fresh $\left(7.01 \mathrm{~g} \mathrm{planta}^{-1}\right)$ and dry matter $\left(2.40 \mathrm{~g} \mathrm{planta}^{-1}\right)$. The mixture of carbonized rice husk + soil or the sand are adequated to be used in the production of mint seedlings in field.
\end{abstract}

Keywords: Mentha arvensis, medicinal plants, cuttings.

\section{(Recebido para publicação em 29 de novembro de 2006; aceito em 22 de novembro de 2007)}

$\mathrm{E}$ spécies de Mentha têm sido investigadas, tanto por suas atividades biológicas como também pelos óleos essenciais produzidos por suas folhas. A espécie Mentha arvensis é uma planta medicinal e aromática cultivada em todo o Brasil, sendo largamente utilizada pelas indústrias químicas, farmacêuticas e de alimentos (Paulus et al., 2005).

Todo material natural ou artificial, puro ou em mistura, que colocado em um recipiente permita a fixação do sistema radicular e possibilite suporte às plantas, pode ser considerado um substrato agrícola (Blanc, 1987). Para possibilitar o crescimento e a atividade do sistema radicular de plantas, entre outras propriedades físicas e químicas, um substrato agrícola deve reter água sem diminuir a disponibilidade de oxigênio para as raízes. O oxigênio é indispensável para a respiração das raízes a fim de suprir a energia necessária à absorção dos nutrientes (Salsac et al., 1987).
O manejo correto e o tipo de substrato agrícola exercem influência significativa na formação do sistema radicular de plantas e, consequentemente, podem interferir no estado nutricional das mesmas (Spurr \& Barnes, 1973). A escolha de substratos pode ser feita com base nas propriedades da matéria prima, tais como: disponibilidade, custo, características físicas, como densidade; características químicas, como pH e condutividade elétrica (Toledo, 1992) e, ainda, em função das exigências da espécie vegetal a ser cultivada (Verdonck et al., 1981). Na produção de mudas de diferentes espécies, a facilidade que o substrato proporciona no momento de retirada da muda do recipiente é outra propriedade importante a ser observada na escolha de um substrato.

As características físicas principalmente relações entre volume de água e ar presentes no substrato, influem na morfologia das raízes adventícias formadas e em suas ramificações (Wilson, 1983;
Bellé, 1990). Diferentes substratos são utilizados como tecnologia para a produção de mudas e para o enraizamento de diferentes espécies vegetais. Em função do tipo de substrato utilizado e do manejo empregado, estacas podem apresentar raízes adventícias desuniformes, refletindo no pegamento e desenvolvimento da planta, após o transplante no campo.

No Rio Grande do Sul, a utilização de solo natural (Gauland, 1997) ou da mistura de solo com areia ainda é prática rotineira dos viveiristas de mudas frutíferas e flores, por sua grande disponibilidade e baixo custo. Porém, estes substratos podem apresentar inconvenientes no crescimento destas plantas, quando utilizados como substrato único, tornando-se necessária a busca de materiais alternativos que permitam melhorar as condições dos substratos utilizados, sem aumentar demasiadamente seu custo.

O substrato organo-mineral

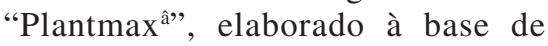


vermiculita expandida e material orgânico, possui macro e micronutrientes necessários ao desenvolvimento inicial das mudas, boas características físicas, boa capacidade de retenção de água e é livre de pragas e doenças (Lopes, 1996).

A casca de arroz carbonizada é outro material que está sendo estudado em mistura de substratos para produção de mudas, por apresentar grande capacidade de drenagem, baixa densidade, $\mathrm{pH}$ levemente alcalino, boa disponibilidade de cálcio e potássio, ausência de nematóides e outros patógenos, além de não necessitar de tratamento químico para esterilização, em função da carbonização (Minami, 1995).

Com relação à utilização da areia na mistura de substratos, Fachinello et al. (1995) citam que também é um material que pode fazer parte do substrato para produção de mudas, por ser de baixo custo, fácil disponibilidade e principalmente por permitir boa drenagem. Contudo, Campos et al. (1986) observaram que a mesma é pobre em nutrientes.

Gomes et al. (1985), relatam que, quando utilizaram terra de subsolo como substrato predominante, o mesmo apresentou dificuldades de drenagem, prejudicando a germinação de sementes, o crescimento e desenvolvimento das mudas de Eucaliptus grandis em tubetes e bandejas de isopor. Entretanto, tal problema foi contornado pela mistura com casca de arroz e bagaço de cana carbonizados (Aguiar et al., 1989).

A forma usual para produção de mudas de menta a campo é a utilização de estolões. Com essa técnica a porcentagem de pegamento é menor e a muda muitas vezes de qualidade inferior, resultando em desuniformidade e menor rendimento de plantas no campo. A geração de novas tecnologias para produção de mudas de menta, envolvendo o uso de substratos, torna-se necessária.

Os estudos de substratos para produção de mudas de menta para o cultivo a campo ainda são escassos. Desta forma, o presente trabalho teve como objetivo avaliar substratos visando a produção de mudas de hortelã para cultivo a campo, pelo método da propagação vegetativa por estaquia.

\section{MATERIAL E MÉTODOS}

Os experimentos foram realizados em duas etapas: produção de mudas em

Tabela 1. Características físicas e químicas dos substratos avaliados para produção de mudas de hortelã quanto à densidade, ao volume de água retida (volume retido) e à capacidade máxima de retenção de água em percentagem de massa úmida (CMR), $\mathrm{pH}$ em água $(\mathrm{pH})$ e à capacidade de troca de cátions (CTC) (chemical and physical characteristics of substrates evaluated for mint seedlings production as the density, volume of storage water (storage volume) and water holding capacity (CMR) in percentage of wet mass, $\mathrm{pH}$ in water $(\mathrm{pH})$ and the cation exchange capacity (CTC)). Santa Maria, UFSM, 2004.

\begin{tabular}{|c|c|c|c|c|c|}
\hline \multicolumn{3}{|c|}{ Características físicas } & \multicolumn{3}{|c|}{ Características químicas } \\
\hline Substratos & $\begin{array}{c}\text { Densidade } \\
\left(\mathrm{kg} \mathrm{m}^{-3}\right)\end{array}$ & $\begin{array}{l}\text { Volume } \\
\text { retido } \\
\left(\mathrm{m}^{3} \mathrm{~m}^{-3}\right)\end{array}$ & $\begin{array}{c}\text { CMR } \\
(\%)\end{array}$ & $\mathrm{pH}$ & $\underset{\mathrm{L}^{-1}}{\text { CTC cmolc }}$ \\
\hline 1 & 414,2 & 0,56 & 55,13 & 5,0 & 31 \\
\hline 2 & 680,9 & 0,45 & 46,2 & 6,3 & 5,3 \\
\hline 3 & 830,17 & 0,35 & 40,73 & 5,6 & 8 \\
\hline Ideal $^{*}$ & $170-1000^{(1)}$ & $0,20-0,80^{(2)}$ & & $\begin{array}{l}6,0-7,0^{(3)} \\
\left.5,2-5,5^{(4)}\right)\end{array}$ & $>12^{(5)}$ \\
\hline
\end{tabular}

* Valores considerados ideais de acordo com: $\left({ }^{1}\right)$ densidade, Grolli (1991); $\left({ }^{2}\right)$ volume (Grolli, 1991); $\left({ }^{3}\right) \mathrm{pH}$, com materiais e mistura de base mineral (Kämpf, 2000); $\left({ }^{4}\right) \mathrm{pH}$, com materiais e mistura de base orgânica (Kämpf, 2000); $\left(^{5}\right)$ CTC (Penningsfeld, 1983). (values considered proper in accordance with: $\left(^{1}\right)$ density Grolli (1991); $\left({ }^{2}\right)$ volume (Grolli, 1991); $\left(^{3}\right) \mathrm{pH}$, with materials and mixture of mineral base (Kämpf, 2000); $\left({ }^{4}\right) \mathrm{pH}$, with materials and mixture of organic base (Kämpf, 2000); ( $\left.{ }^{5}\right)$ CTC (Penningsfeld, 1983)).

tubetes no ambiente protegido e desenvolvimento no solo a campo, de setembro a novembro de 2004, em área experimental da Universidade Federal de Santa Maria (RS).

Avaliaram-se as características físicas e químicas dos substratos (Tabela 1). A densidade, o volume retido de água e a capacidade máxima de retenção de água, foram obtidos conforme Andriolo \& Poerscke (1997). O pH em água foi determinado em potenciômetro, como sugerido por Hoffmann (1970), e a capacidade de troca de cátions (CTC) foi obtida de acordo com metodologia descrita por Tedesco et al.(1995).

A casca de arroz, antes de ser utilizada na formação dos substratos 2 e 3, foi submetida à queima parcial conforme processo descrito por Backes (1998), e o solo de superfície, utilizado na mistura do substrato 2, é classificado como Podzólico vermelho-amarelo, Unidade de Mapeamento São Pedro (Embrapa, 1999).

As plantas matrizes utilizadas na obtenção das estacas foram cultivadas em canteiros, em ambiente de $250 \mathrm{~m}^{2}$, protegido por estrutura de madeira recoberta com polivinilcloreto (PVC) de $250 \mu$ de espessura. As estacas foram retiradas com quatro folhas e aproximadamente $4 \mathrm{~cm}$ de comprimento, do ápice das plantas matrizes. Posteriormente, foram colocadas para enraizar em substrato espuma fenólica, em sistema de berçário constituído de tubos de polipropileno, colocados sobre cavaletes com desnível de $1 \%$ para o escoamento da solução nutritiva para menta, a 50\% de concentração (Paulus, 2004). As mudas, com 10 dias e seis folhas foram transplantadas para tubetes de 142 $\mathrm{mm}$ de altura, $38 \mathrm{~mm}$ de diâmetro e volume de $65 \mathrm{~cm}^{3}$, contendo os diferentes substratos, uma muda/tubete.

A fertirrigação nos tubetes foi com solução nutritiva a 50\% (Paulus, 2004). Permaneceram 45 mudas nos tubetes nos substratos 1, 2 e 3 e 45 mudas, quando estavam com 10 dias e com 14 folhas foram transplantadas ao campo no espaçamento de $0,70 \mathrm{~m}$ entre linhas e 0,30 $m$ entre plantas. As observações foram aos $7,14,21$ e 28 dias após o transplante no campo e nos tubetes nos substratos $1,2 \mathrm{e}$ 3 após colocação das mudas nos substratos em ambiente protegido.

O delineamento experimental foi inteiramente casualizado, com três tratamentos e 15 repetições. Os tratamentos foram 1) Substrato organo-mineral "Plantmax ${ }^{\hat{a} " ; ~ 2) ~ C a s c a ~ d e ~ a r r o z ~+~ s o l o ~}$ (1:1) e 3) Casca de arroz + areia (1:1). Foram avaliados a altura das mudas, número de folhas, índice de mortalidade, fitomassa fresca e seca. A determinação da fitomassa foi realizada aos 35 DAT. As folhas, as hastes e raízes da planta foram separadas e colocadas em estufa com circulação de ar forçada a $65^{\circ} \mathrm{C}$ para obtenção da massa seca. Os dados foram submetidos à análise da 
Tabela 2. Altura de plantas de Mentha arvensis L. produzidas em tubetes com três substratos em ambiente protegido e no solo a campo (height of Mentha arvensis L. plants produced in plastic tubes with three different substrates under protected environment and field cultivation). Santa Maria, UFSM, 2004.

\begin{tabular}{|c|c|c|c|c|c|}
\hline \multirow{2}{*}{ Local } & \multirow{2}{*}{ Substratos } & \multicolumn{4}{|c|}{ Altura $(\mathrm{cm})$} \\
\hline & & 7 DAT & 14 DAT & 21 DAT & 28 DAT \\
\hline \multirow[t]{4}{*}{ A. protegido } & Organo-mineral & $13,00 a^{*}$ & $20,05 \mathrm{a}$ & $22,70 \mathrm{a}$ & $24,6 a$ \\
\hline & C. A. Carb. + solo & $10,50 \mathrm{~b}$ & $17,63 \mathrm{~b}$ & $18,37 \mathrm{~b}$ & $20,63 b$ \\
\hline & C. A. Carb. + areia & $10,30 \mathrm{~b}$ & $16,45 \mathrm{~b}$ & $16,53 \mathrm{~b}$ & $18,45 b$ \\
\hline & C.V.(\%) & 12 & 10 & 13 & 9 \\
\hline \multirow[t]{4}{*}{ Campo } & Organo-mineral & $17,00 a^{*}$ & $23,50 \mathrm{a}$ & $30,00 \mathrm{a}$ & $41,50 \mathrm{a}$ \\
\hline & C. A. Carb. + solo & $14,00 \mathrm{~b}$ & $18,00 \mathrm{~b}$ & $25,00 \mathrm{~b}$ & $34,20 \mathrm{~b}$ \\
\hline & C. A. Carb. + areia & $12,50 \mathrm{~b}$ & $16,70 \mathrm{~b}$ & $22,50 \mathrm{c}$ & $32,70 \mathrm{~b}$ \\
\hline & C.V.(\%) & 14 & 10 & 8 & 11 \\
\hline
\end{tabular}

*Médias seguidas das mesmas letras, nas colunas, não diferem estatisticamente entre si pelo teste de Tukey, a 5\% de probabilidade. (means followed by the same letter in the column do not differ from each other, Tukey's test, $\mathrm{p}<0,05)$.

Tabela 3. Número de folhas por planta de Mentha arvensis L. produzidas em tubetes com três diferentes substratos na fase de produção de mudas e na fase de desenvolvimento no solo, a campo (number of leaves for plant of Mentha arvensis L. produced in plastic tubes with three different substrates in the phase of seedlings production and in field growth). Santa Maria, UFSM, 2004.

\begin{tabular}{|c|c|c|c|c|c|}
\hline \multicolumn{6}{|c|}{ Número de folhas } \\
\hline Local & Substratos & 7 DAT & 14 DAT & 21 DAT & 28 DAT \\
\hline \multirow[t]{4}{*}{ A. protegido } & Organo-mineral & $15,40 a^{*}$ & $16,07 \mathrm{a}$ & 24,45 a & $29,00 \mathrm{a}$ \\
\hline & C. A. Carb. + solo & $13,87 \mathrm{~b}$ & $14,67 \mathrm{~b}$ & $18,27 \mathrm{~b}$ & $22,40 \mathrm{~b}$ \\
\hline & C. A. Carb. + areia & $13,47 \mathrm{~b}$ & $14,13 \mathrm{~b}$ & $15,53 \mathrm{~b}$ & $18,70 \mathrm{~b}$ \\
\hline & C.V.(\%) & 8,32 & 11 & 12 & 14 \\
\hline \multirow[t]{4}{*}{ Campo } & Organo-mineral & $17,00 a^{*}$ & $23,50 \mathrm{a}$ & $30,00 \mathrm{a}$ & $35,02 \mathrm{a}$ \\
\hline & C. A. Carb. + solo & $14,00 \mathrm{~b}$ & $18,00 \mathrm{~b}$ & $25,00 \mathrm{~b}$ & $30,00 \mathrm{~b}$ \\
\hline & C. A. Carb. + areia & $12,50 \mathrm{~b}$ & $16,70 \mathrm{~b}$ & $22,50 \mathrm{c}$ & $25,45 c$ \\
\hline & C.V. $(\%)$ & 12 & 10 & 8 & 11 \\
\hline
\end{tabular}

*Médias seguidas das mesmas letras, nas colunas, não diferem estatisticamente entre si pelo teste de Tukey, a 5\% de probabilidade (means followed by the same letter in the column do not differ from each other, Tukey's test, $\mathrm{p}<0,05$ ).

variância, sendo as médias testadas pelo teste de Tukey a $5 \%$ de probabilidade.

\section{RESULTADOS E DISCUSSÃO}

Para altura das mudas (Tabela 2) aos 7 , 14, 21 e 28 dias após o transplante, na fase de produção de mudas em substrato e na fase de desenvolvimento a campo verificou-se que o melhor tratamento foi o substrato Plantmaxâ. Já os substratos casca de arroz + areia e casca de arroz + solo não diferiram estatisticamente entre si na fase de produção de mudas em substrato.

Com relação às médias dos tratamentos, observou-se evolução no ganho em altura, sendo que na fase inicial obteve-se um ganho inicial de $6,77 \mathrm{~cm} \mathrm{e} \mathrm{4,89} \mathrm{cm}$ aos
(Tabelas 3) aos 7, 14, 21 e 28 dias após transplante, sendo que o substrato Plantmax ${ }^{\hat{a}}$ foi o melhor tratamento. Os tratamentos casca de arroz + areia e casca de arroz + solo não diferiram estatisticamente entre si. Constatou-se acréscimo do número de folhas nos intervalos de tempo avaliados, sendo de 0,76 $\mathrm{cm}$ e $4,13 \mathrm{~cm}$ aos 14 DAT; $4,42 \mathrm{~cm} \mathrm{e}$ $6,27 \mathrm{~cm}$ aos 21 DAT e $3,95 \mathrm{~cm}$ e 4,36 $\mathrm{cm}$ aos 28 DAT no ambiente protegido e no campo, respectivamente.

Schmitz et al. (2002), avaliando as características físicas de diversos substratos, constataram que materiais minerais, como solo e areia, possuem excessiva densidade e reduzida porosidade, sendo deficientes em aeração. Isso foi constatado na análise física dos substratos, onde a densidade do solo foi 1,06 $\mathrm{g} \mathrm{L}^{-1} \mathrm{e}$ da areia foi $1.368 \mathrm{~g} \mathrm{~L}^{-1}$ que proporcionou um reduzido espaço poroso total de $47,7 \%$ e $32,5 \%$, respectivamente.

$\mathrm{Na}$ produção de massa fresca e seca observou-se que o substrato Plantmax ${ }^{\circledR}$ foi superior aos demais tratamentos (Tabela4). As mudas produzidas em substrato Plantmax ${ }^{\text {â }}$ produziram 1,23 g planta $^{-1}$ e 2,43 $\mathrm{g}_{\text {planta }}{ }^{-1}$ a mais que o substrato casca de arroz carbonizada + solo, no ambiente protegido e no campo, respectivamente.

A superioridade do substrato Plantmax ${ }^{\hat{a}}$ observada nos experimentos, foi provavelmente em função de suas características físicas e químicas (Tabela 1), ou seja, menor densidade $\left(414,2 \mathrm{~kg} \mathrm{~m}^{-3}\right)$ que o substrato casca de arroz carbonizada + areia $\left(830,17 \mathrm{~kg} \mathrm{~m}^{-3}\right)$, maior volume retido de água $\left(0,56 \mathrm{~m}^{3} \mathrm{~m}^{-3}\right)$, maior capacidade de armazenamento de água por unidade de volume, o que contribui para a menor oscilação da quantidade de água ofertada para a planta durante o dia, favorecendo assim seu crescimento e desenvolvimento. Com relação às características químicas, o valor mais elevado de CTC (Tabela 1) proporcionou maior capacidade de armazenamento e de fornecimento dos nutrientes às plantas e, mesmo com $\mathrm{pH}$ abaixo de 5,0, foram favorecidos o crescimento e o desenvolvimento das plantas. Já o substrato casca de arroz carbonizada + areia apresentou menor crescimento e desenvolvimento, em função desse substrato ser mais denso, devido à areia apresentar menor capacidade de armazenamento de água por unidade de volume e de massa úmida. Também, devido aos valores de CTC menores do que os da faixa ideal ( $>12$ $\mathrm{cmol}_{\mathrm{c}} \mathrm{L}^{-1}$ ), estabelecida por Penningsfeld 
(1983). Segundo essa especificação, nos demais substratos em que se obtiveram valores de CTC superiores ao mínimo ideal, não se tem problemas para produção de mudas de menta.

Verdonck et al. (1981) afirmam que as características físicas do substrato são as mais importantes, por causa das relações ar-água não poderem sofrer mudanças durante o cultivo. Entre essas, Kämpf (2001) cita a densidade, a porosidade, a disponibilidade de água e de ar do substrato. Para substratos, buscam-se valores de porosidade total entre 0,75 - 0,90 $\mathrm{m}^{3} \mathrm{~m}^{-3}$, para melhor aeração, infiltração de água e drenagem (Kämpf ,2001).

Lira (1990), estudando efeitos de substrato e do superfosfato simples na produção de mudas de limoeiro até repicagem, concluiu que o substrato organo-mineral ("Plantmax $x^{\hat{a}}$ ") proporcionou melhores características de fertilidade e teores de nutrientes na fitomassa seca total das plantas e maior crescimento do limoeiro "cravo", quando comparado com tratamentos à base de um Latossolo Vermelho Amarelo húmico misturado com Latossolo Roxo e vermiculita. Resultados semelhantes foram obtidos por Lopes (1996), em trabalho de propagação sexuada de mudas de maracujazeiro em tubetes, com uso de diferentes substratos e doses de adubação nitrogenada, que concluiu que o substato organo-mineral ("Plantmax") proporcionou melhor desenvolvimento das mudas em comparação com os outros utilizados, com as seguintes composições: a) $70 \%$ de vermiculita $+30 \%$ de terra de subsuperfície; b) $40 \%$ de casca de arroz carbonizada $+30 \%$ de terra de subsuperfície $+30 \%$ de areia.

Considerando os resultados obtidos, o substrato Plantmax ${ }^{\hat{a}}$ pode ser recomendado para produção de mudas de menta pelo método de propagação vegetativa por estaquia. Porém, tanto a mistura casca de arroz + solo ou areia se mostraram apropriados para produção de mudas de menta, pois são materiais de baixo custo, inclusive a casca de arroz é um subproduto abundante da agroindústria arrozeira.

\section{REFERÊNCIAS}

ANDRIOLO JL; POERSCKE PL. 1997. Cultivo do tomateiro em substratos. Santa Maria: UFSM - Centro de Ciências Rurais. 12 p. (Informe Técnico, 2)

AGUIAR IB; VALERI SV; BANZATO DA. 1989 Seleção de componentes de substrato para produção de mudas de Eucaliptus spp. em tubetes. IPEF 41/42: 36-43.

Tabela 4. Massa fresca e seca $\left(\mathrm{g}_{\text {planta }}{ }^{-1}\right)$ de plantas de Mentha arvensis L. produzidas em tubetes com três diferentes substratos na fase de produção de mudas e na fase de desenvolvimento no solo, a campo, obtidas 35 dias após o transplante (fresh and dry mass ( $\mathrm{g} \mathrm{plant}^{-1}$ ) of Mentha arvensis L. plants produced in plastic tubes with three different substrates in the phase of seedlings production and field growth, obtained 35 days after transplanting date). Santa Maria, UFSM, 2004.

\begin{tabular}{llcc}
\hline Local & Substrato & M. fresca $\left(\right.$ g planta $\left.^{-1}\right)$ & M. seca (g planta \\
\hline A. protegido & (g) \\
& Organo-mineral & $7,07 \mathrm{a}^{*}$ & $2,40 \mathrm{a}$ \\
& C. A. Carb. + solo & $2,66 \mathrm{~b}$ & $1,17 \mathrm{~b}$ \\
& C. A. Carb. + areia & $1,89 \mathrm{c}$ & $0,98 \mathrm{~b}$ \\
\hline C.V.(\%) & & 13 & 11 \\
\hline Campo & Organo-mineral & $30,72 \mathrm{a}^{*}$ & $6,68 \mathrm{a}$ \\
& C. A. Carb. + solo & $18,87 \mathrm{~b}$ & $4,25 \mathrm{~b}$ \\
& C. A. Carb. + areia & $16,42 \mathrm{~b}$ & $4,14 \mathrm{~b}$ \\
\hline C.V. $(\%)$ & & 13 & 12 \\
\hline
\end{tabular}

*Médias seguidas das mesmas letras, nas colunas, não diferem estatisticamente pelo teste de Tukey, a 5\% de probabilidade (means followed by the same letter in the column do not differ from each other, Tukey's test, $\mathrm{p}<0,05)$.

BACKES MA. 1989. Composto de lixo urbano como substrato para plantas ornamentais. Porto Alegre: UFRGS. 78p. (Tese mestrado) BELLÉ S. 1990. Uso da turfa "Lagoa dos Patos (Viamão/RS). Porto Alegre: UFRGS. 142p (Tese mestrado).

BLANCD. Les substrats. In: Blanc, M. Ed. 1987.Les cultures hors sol, Paris: INRA, p.9-13.

CAMPOS LAA; SÁ JCA; DEMATÊ MES; VELHO LMLS; VICENTE MEA. 1986. Influência da profundidade de semeadura e substratos no desenvolvimento de sibipiruna (Caesalpina peltophoroides Benth). Científica 14: 101-113.

EMBRAPA Centro Nacional de Pesquisa de Solos (Rio de Janeiro, RJ). 1999. Sistema Brasileiro de Classificação de Solos. Brasília: EMBRAPA - Produção de informação; Rio de Janeiro: EMBRAPA Solos. 26 a ed. p. 209.

FACHINELLO JC; HOFFMANNA; NACHTGAL JC. 1994. Propagação de plantas frutíferas de clima temperado. Pelotas: UFPEL. 179p.

GAULAND DCSP. 1997. Relações hídricas em substratos à base de turfas sob o uso dos condicionadores casca de arroz carbonizada ou queimada. Porto Alegre: UFRGS. 107p. (Tese mestrado).

GOMES JM; COUTO L; PEREIRA AR. 1985. Uso de diferentes substratos na produção de mudas de Eucaliptus grandis em tubetes e em bandejas de isopor. Revista Árvore 9: 58-86.

GROLLI PR. 1991. Composto de lixo domiciliar urbano como condicionador de substratos para plantas arbóreas. Porto Alegre: UFRGS. 125p. (Tese mestrado).

HOFFMANN G. 1970. Verbindliche Methoden zur Untersuchung von TKS und Gärnterischen Erden. Mittelungen der VSLUFA 6: 129-153.

KÄMPF AN. 2000. Seleção de materiais para uso como substrato. In: KÄMPF AN; FERMINO MH; (Eds) Substratos para plantas: à base da produção vegetal em recipientes. Porto Alegre: Gênesis, p.139 - 145 .

KÄMPF AN. 2001. Análise física de substratos para plantas. Viçosa: SBCS. 26: 5-7 (Boletim Informativo).

LIMA NP; BIASI LA; ZANETTE F; NAKASHIMA T. 2003. Produção de mudas por estaquia de duas espécies de guaco. Horticultura Brasileira 21: 106-109.
LIRA LM. 1990. Efeito de substratos e do superfosfato simples no limoeiro (Citrus limonia OSBECK cv. cravo) até a repicagem. Lavras: ESAL. 86p. (Tese mestrado).

LOPES PSN. 1996. Propagação sexuada do maracujazeiro azedo (Passiflora edulis Sims $f$. favicarpa Deg.) em tubetes: efeito da adubação nitrogenada e substratos. Lavras: UFLA. 52p. (Tese mestrado).

MINAMI K. 1995. Produção de mudas de alta qualidade em horticultura. São Paulo: TA. Queiroz, Editor, Ltda. p.128.

PAULUS D; MEDEIROS SLP; SANTOS OS; RIFFEL C; FABBRIN E; PAULUS E. 2005. Substratos na produção hidropônica de mudas de hortelã. Horticultura Brasileira 23: 48-50.

PAULUS D. 2004. Produção, teor e qualidade de óleo essencial de menta em hidroponia e a campo. Santa Maria: UFSM. 63p . (Tese mestrado)

PENNINGSFELD F. 1983. Kultursubstrate fur den Gartenbau, besonders in Deutschland; ein Kritischer Überblick. Plant and Soil 32: 937-944.

SALSAC 1; CHAILLOU S; MOROT-GAUDRY J; LESAINT C; JOLIVET E. 1997. Nitrate and ammonium nutrition in plants. Plant Physiology and Biochemistry 25: 805-812.

SCHMITZ JA; SOUZA PV; KÄMP AN. 2002. Propriedades químicas e físicas de substratos de origem mineral e orgânica para o cultivo de mudas em recipientes. Ciência Rural 32: 937-944.

SPURR SH; BARNES BV. 1973. Forest ecology. New York: The Ronald Press. 571p.

TEDESCO MJ; GIANELLO C; BISSANI CA; BOHNEN H; WOLKWEISS SJ; 1995. Análises de solos, plantas e outros materiais. 2 ed. Porto Alegre: UFRGS/Departamento de Solos, 174p. (Boletim Técnico, 5).

TOLEDO ARM. 1992. Efeito de substratos na formação de mudas de laranjeira (Citrus sinensis (L.) OSBECK cv. "Pera Rio") em vaso. Lavras: ESAL. 88p. (Tese mestrado).

VERDONCK O; VLEESCHAUWER D; DE BOODT M. 1981. The influence of the substrate to plant growth. Acta Horticulturae 126: 251-258.

WILSON GCS. 1983. Use of vermiculite as a growth medium for tomatoes. Acta Horticulturae 150: 283-288. 